Gul Zaman (Chakdara)

Young Han Kang (Daegu)

IL Hyo Jung (Busan)

\title{
DYNAMICS OF A SMOKING MODEL WITH SMOKING DEATH RATE
}

Abstract. In this work, we derive and analyze a smoking model by taking into account hospitalized smokers and smoke quitters of two types: temporary and permanent, which is one of the possible extensions of the giving-up smoking model. Temporary quitters may become smokers again, while permanent quitters, once they quit, never smoke again in the entire life span. The existence and stability of the possible equilibria of the model are examined in terms of a certain threshold condition $\mathcal{R}$, the smoking generation number (the basic reproduction number). Numerical simulations are carried out to investigate the influence of the key parameters on the spread of smoking, to support the analytical conclusion and illustrate possible behavioral scenarios.

1. Introduction. Mathematical models can provide a useful tool to analyze the spread and control of diseases [2, 4, 13, 15].

Castillo-Garsow et al. [5] developed a mathematical model of giving-up smoking and analyzed the dynamical behavior of different smoking individuals. Hoogenveen et al. [10] presented a model that was capable of describing the effects of smoking cessation on morbidity and mortality over time. In their model they described the life course of quitters after smoking cessation, taking into account relapse. The basic model of [5] was extended by Sharomi and Gumel [14] to account for variability in smoking frequency, by introducing the classes of mild and of chain smokers as well as the develop-

2010 Mathematics Subject Classification: 92D25, 49J15, 93D20.

Key words and phrases: population dynamics, mathematical model, stability analysis, numerical simulation.

Received 15 November 2014; revised 15 November 2016 and 31 August 2017.

Published online 12 October 2017. 
ment and the public health impact of smoking-related illnesses. Zaman [16] considered different birth and death rates which ensure that the total population is not constant and derived a model taking into account occasional smokers. He analyzed qualitative behavior and showed both local and global stability with numerical simulations. Zaman et al. [17] established stability and optimal vaccination of the basic model by considering real data. Moreover, non-standard difference techniques for time dependent transmission coefficients in the giving-up smoking model can be found in [18].

In this paper we extend the mathematical model presented in [17] and study its qualitative behavior. Our aim is to derive and analyze the model by taking into account the hospitalized smokers sector and the quitters sector, divided into two subclasses: temporary quitters and permanent quitters. Chain smokers and hospitalized smoking individuals die at different rates because of smoking. Temporary quitters may become smokers again, while permanent quitters, once they quit, never smoke again in the entire life span. In this model, we also assume that some of the smoking individuals may become hospitalized smokers, while some smokers move to the class of temporary quitters and after some time start smoking again. Furthermore, some of the hospitalized smokers stay in this class for a very short time and become smokers again. First, we show the existence and positivity of the proposed model. After a complete stability analysis of the model, we find that there are six equilibria: trivial, smoking-free, and four others corresponding to the presence of smoking. We assume that the birth rate is different from the death rate, which ensures that the total population is not constant. Then, we use the Lyapunov function theory to establish the global stability of the model. Finally, numerical simulations are carried out to investigate the influence of the key parameters on the spread of the disease (smoking), to support the analytical conclusion and illustrate possible behavioral scenarios.

The paper is organized as follows: In Section 2, the model framework is put in place and the model equations are derived. We show the region of biologically feasible solutions, together with their existence and positivity. The smoking-free equilibrium is presented in Section 3. All the possible equilibria of the steady states are provided in Section 4. In the same section, we prove the global stability of the endemic equilibrium. Numerical simulations are carried out to investigate the influence of the key parameters in Section 5. Finally, we present conclusions of this work.

2. Model formulation. The mathematical representation of the givingup smoking model consists of a system of non-linear differential equations with five state variables which is introduced in [16. In our extended model, $P(t)$ denotes the number of potential smokers at time $t ; S(t)$ denotes the 
number of chain smokers at time $t ; H(t)$ denotes the number of hospitalized smokers at time $t ; T(t)$ denotes the number of temporary quitters at time $t$; and $Q(t)$ denotes the number of former smokers that have stopped smoking permanently at time $t$. The total population size at time $t$ is denoted by $N(t)$, so $N(t)=P(t)+S(t)+H(t)+T(t)+Q(t)$. The transfer diagram is depicted in Figure 1. The complete model is described by the following

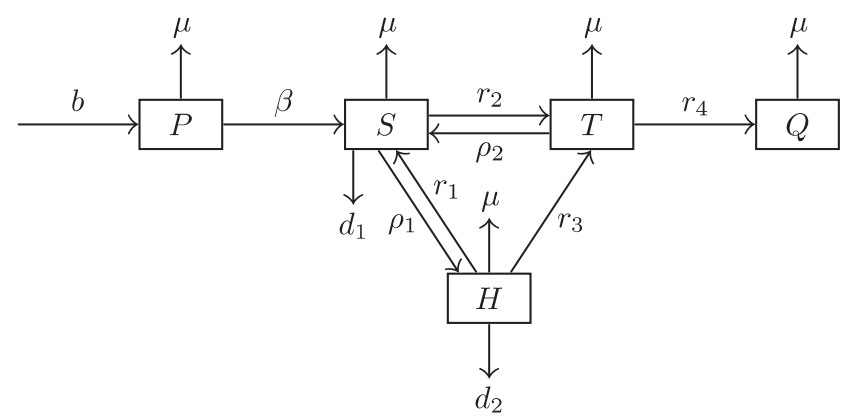

Fig. 1. The diagram represents the mathematical model for smoking population dynamics as defined in the system (1). The boxes represent subpopulations and the arrows represent transitions between the subpopulations, labeled by the parameters of the model.

system of differential equations:

$$
\begin{aligned}
& \frac{d P(t)}{d t}=b N(t)-\beta S(t) P(t)-\mu P(t) \\
& \frac{d S(t)}{d t}=\beta S(t) P(t)-\left(\gamma_{1}+\gamma_{2}+\mu+d_{1}\right) S(t)+\rho_{1} H(t)+\rho_{2} T(t), \\
& \frac{d H(t)}{d t}=\gamma_{1} S(t)-\left(\gamma_{3}+\rho_{1}+\mu+d_{2}\right) H(t), \\
& \frac{d T(t)}{d t}=\gamma_{3} H(t)+\gamma_{2} S(t)-\left(\gamma_{4}+\rho_{2}+\mu\right) T(t), \\
& \frac{d Q(t)}{d t}=\gamma_{4} T(t)-\mu Q(t),
\end{aligned}
$$

where the time-invariant parameters of the model are:

- $b$ : the birth rate (inflow rate) of the population,

- $\mu$ : the natural death rate or death rate related to other diseases besides smoking,

- $\beta$ : social transmission rate from smokers to potential smokers,

- $\gamma_{1}$ : rate at which smoking individuals move to the hospitalized class,

- $\gamma_{2}$ : rate at which smokers become temporary quitters,

- $\gamma_{3}$ : rate at which hospitalized individuals become temporary quitters,

- $\gamma_{4}$ : rate at which temporary quitters become permanent quitters, 
- $d_{1}$ : rate at which smoking individuals die because of smoking,

- $d_{2}$ : rate at which hospitalized smokers die because of smoking,

- $\rho_{1}$ : rate at which hospitalized individuals become smokers,

- $\rho_{2}$ : rate at which temporary quitters relapse.

Moreover, the differential equation for the total population size $N(t)$ takes the form

$$
\frac{d N}{d t}=(b-\mu) N(t)-\left(d_{1} S(t)+d_{2} H(t)\right),
$$

which is derived by adding the five equations of (1). The total population remains constant if $b=\mu$ and $d_{1}=d_{2}=0$ (see for example [12, 4, 16]). In this paper we consider the situation when the total population is not constant, so we may assume that $b<\mu+\min \left\{d_{1}, d_{2}\right\}$.

In order to understand the qualitative behavior of the model, let us consider the set $\Omega$ below with $d=\min \left\{d_{1}, d_{2}\right\}$ and the initial conditions for the system (1) given by

$$
\begin{aligned}
& \Omega=\{(P, S, H, T, Q) \mid 0 \leq P, 0 \leq S, \\
& \quad 0 \leq H, 0 \leq T, 0 \leq Q, d(P+S) \leq N\}, \\
& P(0)=P_{0} \geq 0, \quad S(0)=S_{0} \geq 0, \quad H(0)=H_{0} \geq 0 \\
& T(0)=T_{0} \geq 0, \quad Q(0)=Q_{0} \geq 0 .
\end{aligned}
$$

Now we prove the existence of a non-negative solution of (1) on any finite time interval. In order to do this, we consider the system (1) with initial conditions in (3). We can rewrite (1) in the form

$$
X_{t}=M X+\digamma(X),
$$

where

$$
X=\left[\begin{array}{c}
P(t) \\
S(t) \\
H(t) \\
T(t) \\
Q(t)
\end{array}\right], M=\left[\begin{array}{ccccc}
b-\mu & b & b & b & b \\
0 & -\chi_{1} & \rho_{1} & \rho_{2} & 0 \\
0 & \gamma_{1} & \chi_{2} & 0 & 0 \\
0 & \gamma_{2} & \gamma_{3} & -\chi_{3} & 0 \\
0 & 0 & 0 & \gamma_{4} & -\mu
\end{array}\right], \digamma(X)=\left[\begin{array}{c}
-\beta P(t) S(t) \\
\beta P(t) S(t) \\
0 \\
0 \\
0
\end{array}\right],
$$

with $\chi_{1}=\gamma_{1}+\gamma_{2}+\mu+d_{1}, \chi_{2}=\gamma_{3}+\rho_{1}+\mu+d_{2}, \chi_{3}=\gamma_{4}+\rho_{2}+\mu$, and $X_{t}$ denotes the derivative of $X$ with respect to $t$. The system (4) is a non-linear system with bounded coefficients. We set

$$
G(X)=M X+\digamma(X) .
$$


The second term on the right hand side of (5) satisfies

$$
\left|\digamma\left(X_{1}\right)-\digamma\left(X_{2}\right)\right| \leq \Lambda_{1}\left|P_{1}(t)-P_{2}(t)\right|+\Lambda_{2}\left|S_{1}(t)-S_{2}(t)\right|,
$$

where the positive constants $\Lambda_{1}$ and $\Lambda_{2}$ are independent of $P(t)$ and $S(t)$, respectively. Also we get

$$
\left|G\left(X_{1}\right)-G\left(X_{2}\right)\right| \leq \Lambda\left|X_{1}-X_{2}\right|,
$$

where $\Lambda=\Lambda_{1}+\Lambda_{2}+\|M\|<\infty$. Thus the function $G$ is uniformly Lipschitz continuous. From the feasible region of $\Omega$ and the restriction on $P(t), S(t)$, $H(t), T(t)$ and $Q(t) \geq 0$ we can see that a solution of the system (4) exists (see [3, 6]).

THEOREM 2.1. The solution of the system (1) with initial condition (3) is non-negative for all $t>0$.

Proof. First, we show that $N(t)$ is positive. In order to do this, we recall equation (2):

$$
\frac{d N(t)}{d t}=(b-\mu) N(t)-\left(d_{1} S(t)+d_{2} H(t)\right) .
$$

Since $S(t) \leq N(t)$ and $H(t) \leq N(t)$ for all time $t>0$, we have

$$
\begin{aligned}
\frac{d N(t)}{d t} & \geq(b-\mu) N(t)-\left(d_{1} N(t)+d_{2} N(t)\right) \\
& \geq\left(b-\mu-d_{1}-d_{2}\right) N(t) .
\end{aligned}
$$

By integration from 0 to $t$, we obtain

$$
N(t) \geq N(0) e^{\left(b-\mu-d_{1}-d_{2}\right) t} .
$$

Thus $N(t) \geq 0$ for all $t$.

To show that $P(t)>0$ for all $t>0$, suppose otherwise. Then there exists a time $t_{2}$ such that $P\left(t_{2}\right)=0, P^{\prime}\left(t_{2}\right) \leq 0$ and $S(t) \geq 0, H(t) \geq 0, T(t) \geq 0$ and $Q(t) \geq 0$ for $0 \leq t \leq t_{2}$. However, from the first equation of (1), we have

$$
\frac{d P\left(t_{2}\right)}{d t}=b N\left(t_{2}\right)>0,
$$

which contradicts our supposition that $P^{\prime}\left(t_{2}\right) \leq 0$.

To show that $H(t)>0$, suppose that $H(t)<0$ for some $t$. Let $\Gamma_{1}=$ $\inf \{t: H(t)=0\}$. There exist $\epsilon>0$ such that $0<t_{1}-\Gamma_{1}<\epsilon$ and $H\left(t_{1}\right)<0$, so obviously $d H\left(\Gamma_{1}\right) / d t<0$. But from the third equation of (1) we obtain

$$
\frac{d H\left(\Gamma_{1}\right)}{d t}=\gamma_{1} S\left(\Gamma_{1}\right) \geq 0,
$$

which is a contradiction. Similarly we can prove that $T(t) \geq 0$ for all $t>0$. 
To prove that $Q(t) \geq 0$ for all $t>0$, we can write the fifth equation of (1) as

$$
Q(t)=Q(0) e^{-\mu t}+e^{-\mu t} e^{\int_{0}^{t} e^{\mu x} T(x) d x} .
$$

We have $Q(0)>0$ and $T(t) \geq 0$ for $t>0$, which implies that $Q(t) \geq 0$ for $t>0$.

Let $\alpha(t)=\beta P(t)-\left(\gamma_{1}+\gamma_{2}+\nu+d_{1}\right)$. Then from the second equation of (1) it follows that

$$
\frac{d S(t)}{d t}>S(t) \alpha(t)
$$

because $H(t)>0$ and $T(t) \geq 0$. Since $S(0) \geq 0$, from (6) it follows that $S(t)>0$ for all $t>0$. Hence, we proved that the solution is non-negative for all $t>0$.

The continuity of the right hand side of the system (1) and its derivatives implies that unique solutions exist in the maximal interval. Therefore, solutions enter or stay in $\Omega$ and are eventually bounded and exist for $t \geq 0$, which guarantees that the model is mathematically and epidemiologically well posed [8]. Obviously $\Omega$ is positively invariant and the system (1) is dissipative with the global attractor which is contained in $\Omega$.

3. Smoking-free equilibrium. In this section, we present all the possible equilibria of the model proposed in the previous section. To find the equilibrium points, we set the right hand side of each of the five differential equations in (1) to zero, obtaining the equations

$$
\begin{aligned}
& b N(t)-\beta S(t) P(t)-\mu P(t)=0, \\
& \beta S(t) P(t)-\chi_{1} S(t)+\rho_{1} H(t)+\rho_{2} T(t)=0, \\
& \gamma_{1} S(t)-\chi_{2} H(t)=0, \\
& \gamma_{3} H(t)+\gamma_{2} S(t)-\chi_{3} T(t)=0, \\
& \gamma_{4} T(t)-\mu Q(t)=0 .
\end{aligned}
$$

To obtain the equilibrium of the total population size, we set the right hand side of (2) to zero:

$$
(b-\mu) N(t)-\left(d_{1} S(t)+d_{2} H(t)\right)=0 .
$$

After some manipulation and using the system (7) and equation (8) we find that the unique positive epidemic equilibrium of the system (1) is given by 


$$
\begin{aligned}
P^{\star} & =\frac{1}{\beta} \frac{\chi_{1} \chi_{2} \chi_{3}-\rho_{1} \gamma_{1} \chi_{3}-\rho_{2}\left(\gamma_{1} \gamma_{2}+\gamma_{2} \chi_{2}\right)}{\chi_{2} \chi_{3}}, \\
S^{\star} & =\frac{\mu(b-\mu)\left(\rho_{1} \gamma_{1} \chi_{3}+\rho_{2}\left(\gamma_{1} \gamma_{2}+\gamma_{2} \chi_{2}\right)\right)}{\beta \chi_{3}\left(d_{1} \chi_{2}+\gamma_{1} d_{2}-\beta(b-\mu) \chi_{2} P^{\star}\right)}[\mathcal{R}-1], \\
H^{\star} & =\frac{\gamma_{1}}{\chi_{2}} S^{\star}, \\
T^{\star} & =\frac{\gamma_{1} \gamma_{3}+\gamma_{2} \chi_{2}}{\chi_{2} \chi_{3}} S^{\star}, \\
Q^{\star} & =\frac{\gamma_{4}\left(\gamma_{1} \gamma_{3}+\gamma_{2} \chi_{2}\right)}{\mu\left(\chi_{2} \chi_{3}\right)} S^{\star}, \\
N^{\star} & =\frac{d_{1} \chi_{2}+d_{2} \gamma_{1}}{b \chi_{2}} S^{\star},
\end{aligned}
$$

where $\mathcal{R}=\chi_{1} \chi_{2} \chi_{3} /\left(\rho_{1} \gamma_{1} \chi_{3}+\rho_{2} \gamma_{1} \gamma_{2}+\gamma_{2} \rho_{2} \chi_{2}\right)$ represents the smoking generation number (basic reproductive number). It measures the average number of new smokers generated by a single smoker in a population of potential smokers. In epidemic models, $\mathcal{R}$ represents the number of secondary infections caused by a typical newly infected individual entering the population at the disease-free equilibrium during his or her entire infectious period. So in our proposed model when $\mathcal{R}<1$, there is no smoker in the community, while for $\mathcal{R}>1$, smoking individuals exist.

The points $E_{0}=(0,0,0,0,0)$ and $E_{s}=(N, 0,0,0,0)$ are the trivial equilibrium and the smoking-free equilibrium of the system (1); they exists for all non-negative values of the parameters used in the model. Any equilibrium in $\Omega$ corresponding to the disease (smoking) being endemic is called an endemic equilibrium. Apart from these, there are four more different equilibria of (1):

- Hospitalized-free and quitter-free equilibrium state: $E_{2}=\left(P_{2}, S_{2}, 0,0,0\right)$, where $P_{2}=\mu+d_{1} / \beta, S_{2}=\left(b\left(\mu+d_{1}\right) / \beta\left(b-d_{1}-\mu\right)\right)[\mu / b-1]$.

- Temporary and permanent quitter-free equilibrium state: $E_{3}=\left(P_{3}, S_{3}\right.$, $\left.H_{3}, 0,0\right)$, where

$$
\begin{aligned}
P_{3} & =\frac{\gamma_{1}\left(\mu+d_{2}\right)+\left(\mu+d_{1}\right)\left(\rho_{1}+\mu+d_{2}\right)}{\beta\left(\rho_{1}+\mu+d_{2}\right)}, \\
S_{3} & =\frac{b\left(\gamma_{1}\left(\mu+d_{2}\right)+\left(\mu+d_{1}\right)\left(\rho_{1}+\mu+d_{2}\right)\right)}{\beta\left(\left(\rho_{1}+\mu+d_{2}\right)+b \gamma_{1}-\left(\gamma_{1}\left(\mu+d_{2}\right)+\left(\mu+d_{1}\right)\left(\rho_{1}+\mu+d_{2}\right)\right)\right)}[\mu / b-1], \\
H_{3} & =\frac{\gamma_{1}}{\left(\rho_{1}+\mu+d_{2}\right)} S_{3} .
\end{aligned}
$$

- Hospitalized-free equilibrium state: $E_{4}=\left(P_{4}, S_{4}, 0, T_{4}, Q_{4}\right)$, where

$$
P_{4}=\frac{\gamma_{2}\left(\gamma_{4}+\mu\right)+\left(\mu+d_{1}\right)\left(\rho_{2}+\gamma_{4}+\mu\right)}{\beta\left(\gamma_{4}+\rho_{2}+\mu\right)},
$$




$$
\begin{aligned}
S_{4} & =\frac{b \mu\left(\gamma_{2}\left(\gamma_{4}+\mu\right)+\left(\mu+d_{1}\right)\left(\rho_{2}+\gamma_{4}+\mu\right)\right)}{\beta\left(\mu\left(\rho_{2}+\gamma_{4}+\mu\right)\left(b-\mu-d_{1}\right)+\gamma_{2}(b-\mu)\left(\mu+\gamma_{4}\right)\right)}[\mu / b-1], \\
T_{4} & =\frac{\gamma_{2}}{\left(\gamma_{4}+\rho_{2}+\mu\right)} S_{4}, \\
Q_{4} & =\frac{\gamma_{2} \gamma_{4}}{\mu\left(\gamma_{4}+\rho_{2}+\mu\right)} S_{4},
\end{aligned}
$$

- Permanent quitter-free equilibrium state: $E_{5}=\left(P_{5}, S_{5}, H_{5}, T_{5}, 0\right)$, where $P_{5}=P^{\star}, S_{5}=S^{\star}, H_{5}=H^{\star}$ and $T_{5}=T^{\star}$.

4. Local and global stability analysis. In this section, we analyze both local and global stability of the model including the endemic equilibrium.

REMARK. The Jacobian matrix around the trivial equilibrium $E_{0}=$ $(0,0,0,0,0)$ is

$$
J_{0}=\left[\begin{array}{ccccc}
-\mu & 0 & 0 & 0 & 0 \\
0 & -\chi_{1} & \rho_{1} & \rho_{2} & 0 \\
0 & \gamma_{1} & -\chi_{2} & 0 & 0 \\
0 & \gamma_{2} & \gamma_{3} & -\chi_{3} & 0 \\
0 & 0 & 0 & -\gamma_{4} & -\mu
\end{array}\right],
$$

and because there is only one non-zero element in the first column and in the fifth column, and they are both negative and equal, we may reduce the question of whether the eigenvalues of this matrix have negative real part to the same question for the $3 \times 3$ matrix

$$
J_{0}^{*}=\left[\begin{array}{ccc}
-\chi_{1} & \rho_{1} & \rho_{2} \\
\gamma_{1} & -\chi_{2} & 0 \\
\gamma_{2} & \gamma_{3} & -\chi_{3}
\end{array}\right] .
$$

The characteristic polynomial of the latter is

$$
\psi(x)=x^{3}+c_{1} x^{2}+c_{2} x+c_{3},
$$

where $c_{1}=\chi_{1}+\chi_{2}+\chi_{3}, c_{2}=\chi_{1} \chi_{2}+\chi_{1} \chi_{3}+\chi_{2} \chi_{3}-\gamma_{2} \rho_{2}$ and $c_{3}=\chi_{1} \chi_{2} \chi_{3}-$ $\rho_{2} \gamma_{1} \gamma_{3}-\rho_{2} \gamma_{2} \chi_{2}$. Therefore by the Routh-Hurwitz criterion the roots of the polynomial $\psi(x)$ have negative real part when $\chi_{1} \chi_{2}+\chi_{1} \chi_{3}+\chi_{2} \chi_{3}>\gamma_{2} \rho_{2}$ and $\rho_{2} \gamma_{1} \gamma_{3}+\rho_{2} \gamma_{2} \chi_{2}<\chi_{1} \chi_{2} \chi_{3}$, and then the system is asymptotically stable. Thus the trivial equilibrium is locally stable if the parameter values satisfy the above restriction.

THEOREM 4.1. The equilibrium $E_{1}=(1,0,0,0,0)$ is a locally stable smoker-free equilibrium if and only if $\beta<\gamma_{2}+d_{1}+\mu$. Otherwise $E_{1}$ is an unstable smoker-free equilibrium. 
Proof. The local stability of this equilibrium solution can be examined by linearizing the model (1) around $E_{1}=(1,0,0,0,0)$, which gives the Jacobian matrix

$$
J_{1}=\left[\begin{array}{ccccc}
-\mu & -\beta & 0 & 0 & 0 \\
0 & \beta-\chi_{1} & \rho_{1} & \rho_{2} & 0 \\
0 & \gamma_{1} & -\chi_{2} & 0 & 0 \\
0 & \gamma_{2} & \gamma_{3} & -\chi_{3} & 0 \\
0 & 0 & 0 & -\gamma_{4} & -\mu
\end{array}\right] .
$$

Similarly there is only one non-zero element in the first column and in the fifth column, and they are both negative and equal, so we may reduce the question of whether the eigenvalues of this matrix have negative real part to the same question for the $3 \times 3$ matrix

$$
J_{0}^{*}=\left[\begin{array}{ccc}
\beta-\chi_{1} & \rho_{1} & \rho_{2} \\
\gamma_{1} & -\chi_{2} & 0 \\
\gamma_{2} & \gamma_{3} & -\chi_{3}
\end{array}\right] .
$$

Thus, all the roots have negative real part when $\beta<\gamma_{2}+d_{1}+\mu$, and then the smoker-free equilibrium is locally asymptotically stable.

Theorem 4.2. For $\mathcal{R}>1$ and $\left(d_{1} \chi_{2}+\gamma_{1} d_{2}+\beta \mu \chi_{1} \chi_{2}^{2} \chi_{3}\right) / \beta \chi_{1} \chi_{2}^{2} \chi_{3}>b$, the unique positive epidemic equilibrium of the system (1) is locally asymptotically stable.

Proof. The Jacobian matrix at the unique positive epidemic equilibrium is

$$
J=\left[\begin{array}{ccccc}
-\beta S^{\star}-\mu & -\beta P^{\star} & 0 & 0 & 0 \\
\beta S^{\star} & \beta P^{\star}-\chi_{1} & \rho_{1} & \rho_{2} & 0 \\
0 & \gamma_{1} & -\chi_{2} & 0 & 0 \\
0 & \gamma_{2} & \gamma_{3} & -\chi_{3} & 0 \\
0 & 0 & 0 & -\gamma_{4} & -\mu
\end{array}\right] .
$$

As there is only one non-zero element $-\mu$ in the fifth column, we may reduce the question of whether the eigenvalues of this matrix have negative real part to the same question for the $4 \times 4$ matrix

$$
J^{\star}=\left[\begin{array}{cccc}
-\beta S^{\star}-\mu & -\beta P^{\star} & 0 & 0 \\
\beta S^{\star} & \beta P^{\star}-\chi_{1} & \rho_{1} & \rho_{2} \\
0 & \gamma_{1} & -\chi_{2} & 0 \\
0 & \gamma_{2} & \gamma_{3} & -\chi_{3}
\end{array}\right] .
$$


The characteristic polynomial becomes

$$
\Psi(x)=x^{4}+a_{1} x^{3}+a_{2} x^{2}+a_{3} x+a_{4},
$$

where the coefficients $a_{i}$ for $i=1,2,3,4$ are given by

$$
\begin{aligned}
a_{1}= & \chi_{1}+\chi_{2}+\chi_{3}+\mu+\beta\left(S^{\star}-P^{\star}\right), \\
a_{2}= & \left(\chi_{1}+\chi_{2}+\chi_{3}-\beta P^{\star}\right)\left(\beta S^{\star}+\mu\right)+\rho_{1} \gamma_{2}+\rho_{2} \gamma_{2}+\chi_{1} \chi_{2}+\chi_{1} \chi_{3}+\chi_{2} \chi_{3} \\
& -\beta\left(\chi_{2}+\chi_{3}\right) P^{\star}+\beta^{2} P^{\star} S^{\star}, \\
a_{3}= & \left(\chi_{1} \chi_{2}+\chi_{1} \chi_{3}+\chi_{2} \chi_{3}-\beta\left(\chi_{2}+\chi_{3}\right) P^{\star}\right)\left(\beta S^{\star}+\mu\right)+\rho_{1} \gamma_{2}+\rho_{2} \gamma_{2}+\beta S^{\star}+\mu \\
& +\chi_{1} \chi_{2} \chi_{3}-\beta \chi_{2} \chi_{3} P^{\star}+\rho_{1} \gamma_{1} \chi_{3}-\rho_{2} \gamma_{1} \gamma_{3}-\rho_{2} \gamma_{2} \chi_{2}+\beta^{2} P^{\star} S^{\star}\left(\chi_{2}+\chi_{3}\right), \\
a_{4}= & \left(\chi_{1} \chi_{2} \chi_{3}-\beta \chi_{1} \chi_{3} P^{\star}+\rho_{1} \gamma_{1} \chi_{3}-\rho_{2} \gamma_{1} \gamma_{3}-\rho_{2} \gamma_{2} \chi_{2}\right)\left(\beta S^{\star}+\mu\right) \\
& +\beta^{2} P^{\star} S^{\star} \chi_{2} \chi_{3} .
\end{aligned}
$$

The equilibrium state is asymptotically stable by the Routh-Hurwitz criterion if $a_{1}, a_{3}, a_{4}>0$ and $a_{1} a_{2} a_{3}>a_{3}^{2}+a_{1}^{2} a_{4}$ [1]. Thus the roots of $\Psi(x)$ have negative real parts when $\left(d_{1} \chi_{2}+\gamma_{1} d_{2}+\beta \mu \chi_{1} \chi_{2}^{2} \chi_{3}\right) / \beta \chi_{1} \chi_{2}^{2} \chi_{3}>b$, and then the system is asymptotically stable.

TheOrem 4.3. The unique positive epidemic equilibrium of the system (1) exists for $\mathcal{R}>1$ and is globally asymptotically stable if $d_{2} / N>b-\mu$.

Proof. We prove the global stability by constructing a suitable Lyapunov function:

$$
L_{1}(t)=\frac{N^{\star}}{b-\mu} \log N(t)+\frac{1}{2}\left(H(t)-H^{\star}\right)^{2} .
$$

Differentiating $L_{1}$ along the system (1) we obtain

$$
\begin{aligned}
L_{1}^{\prime}(t)= & \frac{N^{\star}}{(b-\mu) N(t)} N^{\prime}(t)+\left(H(t)-H^{\star}\right) H^{\prime}(t) \\
= & \frac{N^{\star}}{(b-\mu) N(t)}\left((b-u) N(t)-d_{1} S(t)-d_{2} H(t)\right) \\
& +\left(H(t)-H^{\star}\right)\left(\gamma_{1} S(t)-\chi_{2} H(t)\right) \\
= & -\left[\left(\frac{d_{2}}{(b-\mu) N(t)}-1\right) d_{1} S^{\star}+\left(\frac{d_{2}}{(b-\mu) N}-1\right) d_{2} H^{\star}+\chi_{2} H(t)\right. \\
& \left.+\left(\left(d_{1}^{2} \chi_{2}+d_{1} d_{2}+\gamma_{1} N(t)\right) \frac{S^{\star}}{\chi_{2} N}-\gamma_{1} H(t)\right) S(t)\right] .
\end{aligned}
$$

Since $N(t) \geq H(t)$ and $H(t) \geq H^{\star}$ for all $t>0$, we obtain $L_{1}^{\prime}<0$ if $d_{2} / N>b-\mu$. Also, $L_{1}^{\prime}=0$ if $N(t)=0$. Therefore, by the Lasalle Invariance Principle [6] every solution of (1) with the initial conditions approaches $E_{1}$ as $t \rightarrow \infty$. Thus the unique positive epidemic equilibrium of (1) is globally asymptotically stable. 
5. Numerical simulation. After having shown the stability of the model at the equilibrium point theoretically, we verified this result by doing some simulations. We carried out numerical simulations using MATLAB to illustrate the dynamics of the system. The world population grows in such a way that the number or rate of births exceeds the number or rate of deaths. For example, in 2000 there were 14 births per 1,000 people and 9 deaths per 1,000 people [9]. Therefore, we consider $b=0.0014$ and $\mu=0.009$ to be the birth and death rate, respectively. For numerical simulations of (1) we use different parameter values represented in Table 1 for all possible equilibria. Furthermore, we investigated three different scenarios corresponding to different subclasses and different initial numbers of infectious individuals.

Table 1. Simulation results for possible equilibria

\begin{tabular}{lcccccccccccc}
\hline \multicolumn{1}{c}{$b$} & $\mu$ & $\beta$ & $\gamma_{1}$ & $\gamma_{2}$ & $\gamma_{3}$ & $\gamma_{4}$ & $\rho_{1}$ & $\rho_{2}$ & $d_{1}$ & $d_{2}$ & Results \\
\hline 0.0136 & 0.0136 & 0.024 & 0 & 0 & 0 & 0 & 0 & 0 & 0 & 0 & $E_{2}$ \\
0.0136 & 0.0031 & 0.024 & 0.00015 & 0 & 0 & 0 & 0.002 & 0 & 0.00037 & 0 & $E_{3}$ \\
0.0 .136 & 0.0031 & 0.024 & 0.00015 & 0 & 0.2 & 0 & 0.002 & 0 & 00037 & 0 & $E_{4}$ \\
0.0136 & 0.0031 & 0.024 & 0.00015 & 0.0 & 0.2 & 0 & 0.12 & 0.012 & 0.025 & 0.012 & $E_{5}$ \\
0.024 & 0.021 & 0.014 & 0.006 & 0.031 & 0.012 & 0.023 & 0.012 & 0.08 & 0.025 & 0.021 & $E^{*}$ \\
\hline
\end{tabular}

SCEnario 1. We considered the real data presented in [17, 7] such that $P(0)=153, S(0)=79$, and $Q(0)=68$ and assumed that there are no hospitalized individuals or temporary quitters. The reasonable parameter values $\beta=0.0024, b=\mu=0.0136$ and $\gamma_{2}=\gamma_{4}=0.00015$ are considered for numerical simulation represented in Figure 2. This figure shows that if the value of the interaction term $\beta$ increases, the population of smoking individuals increases and that of potential smokers decreases.

SCenario 2. We considered the same real data for $P(0)=153, S(0)=79$, and we assumed that smokers are divided into two classes: smokers and hospitalized smokers, with $S(0)>H(0)$, but there are no temporary quitters. Korean Medical Insurance Corporation gave cancer mortality rates of 4.0/100,000 in never smokers, of 7.0 in former smokers, and of 12.4 in current smokers [11]. Here, we assumed that during any smoking cessation period all hospitalized smokers are considered as former smokers. Therefore, the (smoking) death rate $d_{1}=0.00014$ and death rate $d_{2}=0.00005$ during smoking cessation time with other parameter values $\beta=0.0024$, $b=0.0136, \mu=0.0031$, and $\gamma_{2}=0.00015$ are considered for numerical simulation to obtain the temporary and permanent quitter-free equilibrium state $E_{3}$ represented in Figure 3. In Figure 3, we plot the potential smokers, chain smokers and hospitalized smokers. The population of potential smokers sharply decreases from the beginning because the maximum number of 


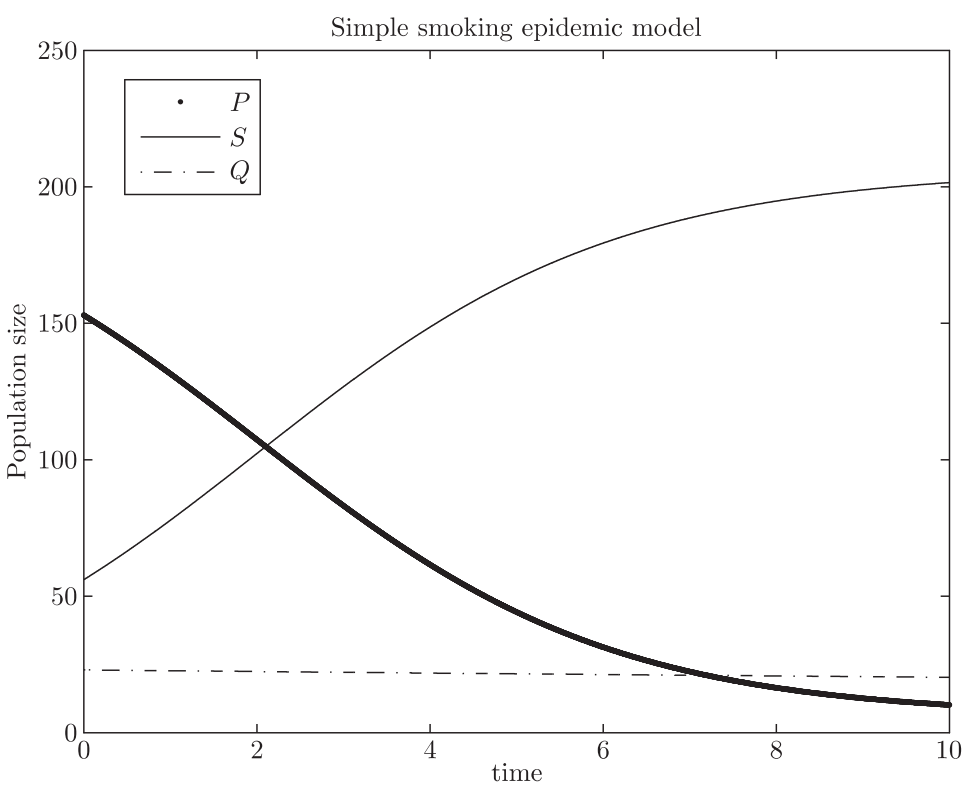

Fig. 2. Potential smokers, smokers and quitters

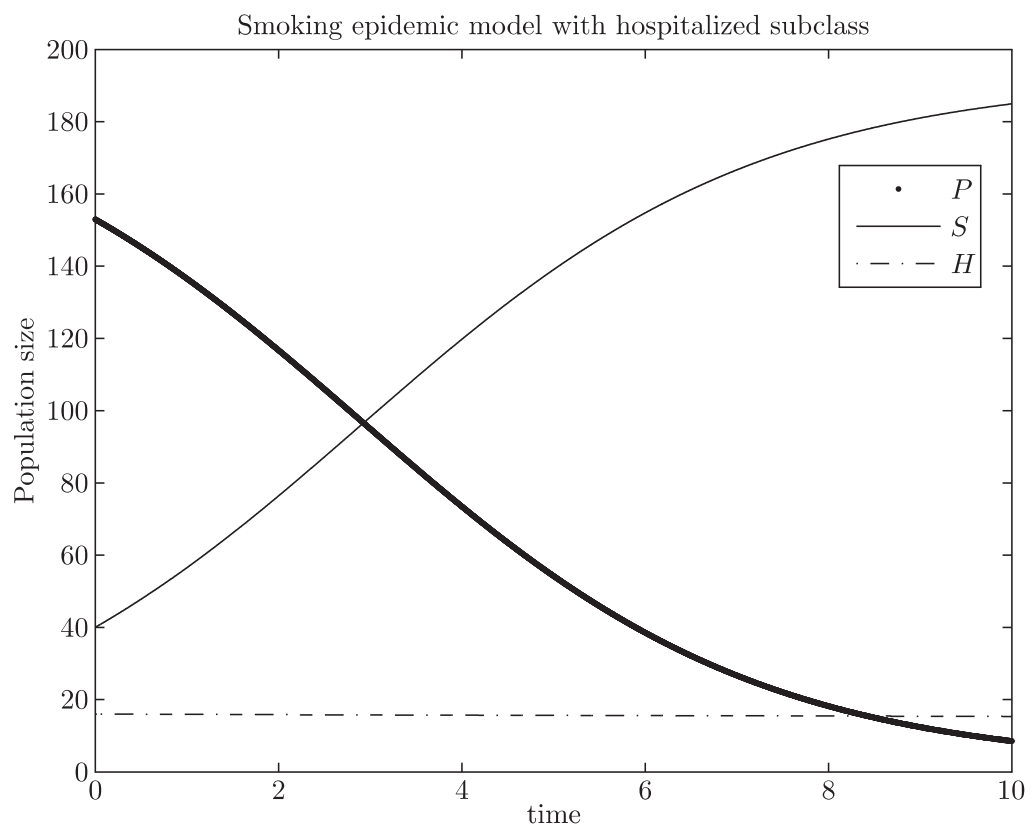

Fig. 3. Potential smokers, smokers and hospitalized smokers

smoking individuals occurs within the first few days. This shows that in the equilibrium state $E_{3}$ some of the temporary quitters have a hard time keep- 
ing up non-smoking. Their motivation has reduced. There may be different reasons to start smoking again. For example, if they constantly see other smokers, or they miss their cigarette in certain situations, or when drastic events happen that cause stress.

SCEnARIo 3. In this scenario we assumed that all five subclasses exist. The real data for potential smokers were $P(0)=153$, for smokers $S(0)=79$, and for quitters $Q(0)=68$. The smokers are divided into two classes: chain smokers and hospitalized smokers, with $S(0)>H(0)$, and quitters are divided into temporary quitters and permanent quitters, with $T(0)+Q(0)=68$. The parameter values $\beta=0.014, b=0.024, \mu=0.021$, $\gamma_{1}=0.006, \gamma_{2}=0.006, \gamma_{3}=0.031, \gamma_{4}=0.00015, \rho_{1}=0.012 \rho_{2}=0.08$ $d_{1}=0.025$ and $d_{2}=0.021$ are considered and the numerical simulation is represented in Figure 4. The population of potential smokers sharply

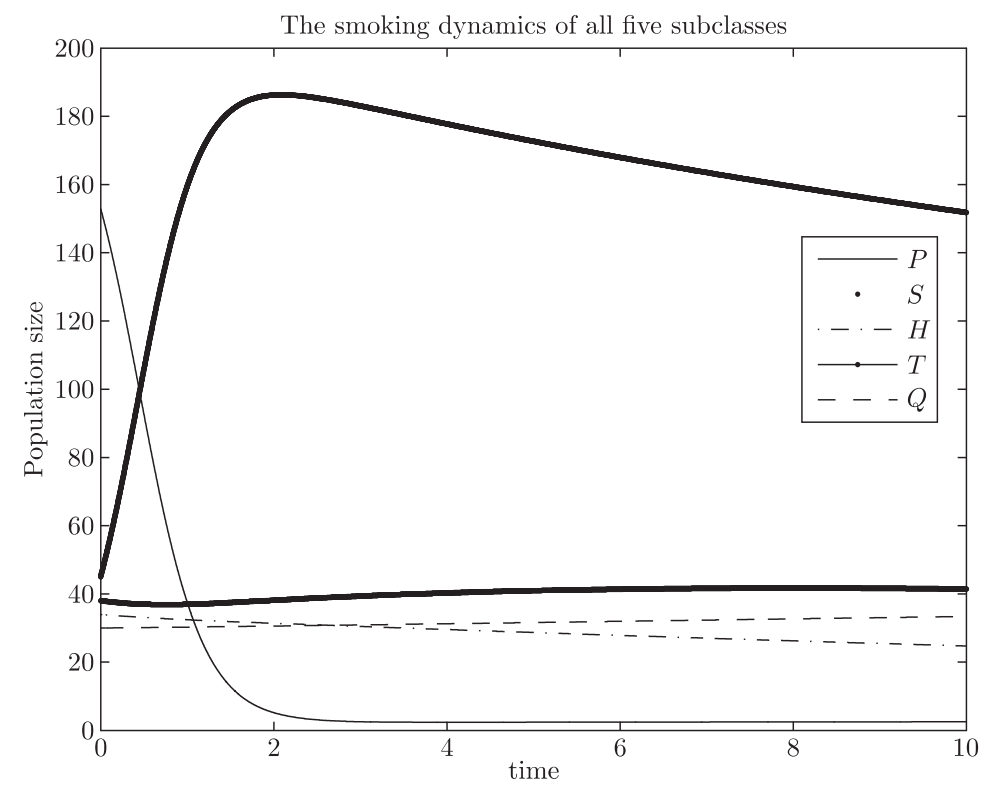

Fig. 4. Potential smokers, smokers, hospitalized smokers, temporary quitters and permanent quitters

decreases from the beginning because the maximum number of smoking individuals occurs within the first few days. This shows that the numbers of temporary and permanent quitters slightly increase, while the number of hospitalized smokers decreases. Their motivation to quit smoking through treatment or education campaign may have reduced.

In this work we used five different sets of parameters for the equilibria of the dynamical system (1). Simulations with different sets of parameter values 
can be used to obtain a sampling of possible behaviors of the dynamical system.

6. Conclusion. In this paper we investigated possible extensions of the giving-up smoking model in a community. We derived the model taking into account hospitalized smokers and quitters of two types: temporary and permanent. We proved the existence and positivity of all individuals in the system. From the analysis of our proposed model we deduced that there are six equilibria: trivial and smoking-free equilibria and four others. Then we established the global stability of the model by Lyapunov function theory. Finally, we estimated the parameters that characterize the natural history of the disease and presented numerical simulations. In fact, we believe that the hospitalized smokers sector and two subclasses of quitters introduced in this paper will help to understand the smoking problem more easily. One future task is the introduction of optimal control programs in the smoking dynamics to see how this would affect the evolution of the spread of smoking.

\section{References}

[1] L. J. S. Allen, An Introduction to Mathematical Biology, Pearson Prentice Hall, 2007.

[2] R. M. Anderson and R. M. May, Infectious Diseases of Humans, Dynamics and Control, Oxford Univ. Press, Oxford, 1991.

[3] G. Birkhoff and G.-C. Rota, Ordinary Differential Equations, 4th ed., Wiley, New York, 1989.

[4] F. Brauer and C. Castillo-Chavez, Mathematical Models in Population Biology and Epidemiology, Springer, New York, 2001.

[5] C. Castillo-Garsow, G. Jordán-Salivia and A. Rodriguez Herrera, Mathematical models for the dynamics of tobacco use, recovery, and relapse, Technical Report Series BU-1505-M, Cornell Univ., 2000.

[6] J. K. Hale, Ordinary Differential Equations, Wiley, New York, 1969.

[7] O. K. Ham, Stages and processes of smoking cessation among adolescents, West J. Nurs. Res. 29 (2007), 301-315.

[8] H. W. Hethcote, The Mathematics of Infectious Diseases, SIAM Rev. 42 (2000), 599-653.

[9] History of Global Population Growth, http://www.deathreference.com/Nu-Pu/Population-Growth.html

[10] R. T. Hoogenveen, P. H. M. van Baal, H. C. Boshuizen and T. L. Feenstra, Dynamic effects of smoking cessation on disease incidence, mortality and quality of life: The role of time since cessation, Cost Eff. Reso. Alloc. 6 (2008), 1-15.

[11] S. H. Jee, J. M. Samet, H. Ohrr, J. H. Kim and I. S. Kim, Smoking and cancer risk in Korean men and women, Cancer Causes Control 15 (2004), 341-348.

[12] W. O. Kermack and A. G. McKendrick, Contribution to the mathematical theory of epidemics, Proc. Roy. Soc. London A 115 (1927), 700-721.

[13] J. D. Murray, Mathematical Biology 2: Spatial Models and Biomedical Applications, 3rd ed., Springer, 2003. 
[14] O. Sharomi and A. B. Gumel, Curtailing smoking dynamics: A mathematical modeling approach, Appl. Math. Comput. 195 (2008), 475-499.

[15] M. Songa, W. Ma and Y. Takeuchib, Permanence of a delayed SIR epidemic model with density dependent birth rate, J. Comput. Appl. Math. 201 (2007), 389-394.

[16] G. Zaman, Qualitative behavior of giving up smoking model, Bull. Malays. Math. Sci. Soc. 34 (2011), 403-415.

[17] G. Zaman, Y. H. Kang and I. H. Jung, Stability and optimal vaccination of an SIR epidemic model, BioSystems 93 (2008), 240-249.

[18] G. Zaman and S. Islam, A non-standard numerical method for a giving-up smoking model, Nonlinear Sci. Lett. A 1 (2010), 397-402.

Gul Zaman

Department of Mathematics

University of Malakand

Chakdara, Dir Lower

Khyber Pakhtunkhawa, Pakistan

E-mail: gzaman@uom.edu.pk

Il Hyo Jung

Department of Mathematics

Pusan National University

Busan 609-735, South Korea

E-mail: ilhjung@pusan.ac.kr
Young Han Kang

Institute of Liberal Education

Catholic University of Daegu Gyeongsan-Si

Gyeongbuk 712-702, South Korea

E-mail: yonghann@cu.ac.kr 
